\title{
Pathogenesis
}

Digestive

Diseases

DOI: $\underline{10.1159 / 000335699}$

\section{Abnormalities of Neuromuscular Anatomy in Diverticular Disease}

\author{
Martina Böttner Thilo Wedel \\ Department of Anatomy, Christian-Albrechts University of Kiel, Kiel, Germany
}

\section{Key Words}

Pathogenesis • Enteric nervous system • Enteric neuropathy • Enteric myopathy

\begin{abstract}
The pathogenesis of diverticular disease is still poorly understood and considered to be multifactorial. Whereas classical pathogenetic concepts have focused on risk factors including increasing age, low-fiber diet and connective tissue disorders, novel concepts take into account that patients with diverticular disease exhibit disturbed intestinal motility patterns (that may result in functional obstruction and painful sensations) therefore postulating an underlying enteric neuro-/myopathy. Recent studies including quantitative evaluations of the enteric nervous system (ENS) in diverticular disease yielded hypoganglionic conditions of both myenteric and submucosal plexus as well as a nerve tissue remodeling in chronic diverticular disease. The disturbed neuromuscular communication was proven by demonstrating alterations in several enteric neurotransmitter systems, exemplified for the cholinergic, serotonergic, nitrergic system as well as for vasointestinal peptide, galanin and tachykinins. Novel lines of evidence have added the involvement of neurotrophic factors such as glial cell line-derived neurotrophic factor which is supposed to regulate ENS development and maintenance and which is downregulated in patients with diverticular disease. Consistent with the hypothesis of an enteric myopathy, deficits in smooth muscle integrity and composi-
\end{abstract}

tion such as hypertrophy, fibrotic transformation and gene expression deficits could be delineated. Taken together, the structural and functional findings on alterations of the ENS and the enteric musculature in diverticular disease provide evidence to strengthen the hypothesis that an enteric neuro-/myopathy may contribute to the development of colonic diverticula and the generation of symptoms in the course of the disease.

Copyright $\odot 2012$ S. Karger AG, Basel

\section{Introduction}

Diverticular disease is the most common morphological abnormality of the colon defined by multiple herniations of the mucosal and submucosal layers through the colonic wall (pseudodiverticula). Due to its high prevalence especially in the elderly and the associated complications, diverticular disease represents the 5th most important gastrointestinal disease in western countries in terms of direct and indirect costs with a mortality rate of 2.5 per 100,000 population per year [1].

In spite of the worldwide distribution of diverticular disease and its socioeconomic impact, the pathogenesis is not yet fully clarified and the etiology is considered to be multifactorial. Factors which have been shown to favor the formation of colonic diverticula include increasing age, low fiber diet, and structural as well as functional alterations of the colonic wall.

PD Dr. rer. nat. Martina Böttne

Department of Anatomy, Christian-Albrechts University of Kiel

Otto-Hahn-Platz 8, DE-24118 Kiel (Germany)

Tel. +49431 8802430

E-Mail m.boettner@anat.uni-kiel.de 


\section{Established Etiological Factors}

\section{Increasing Age}

One well-established factor triggering the development of diverticula is increasing age [2-4]. Whereas this condition is uncommon under the age of 40 , the prevalence increases rapidly from approximately $20 \%$ at the age of 50 to around $60 \%$ at the age of 70 . Although advancing age is obviously associated with diverticular disease, this association may be ostensible, as it is not the age per se but the prolonged time course during which the colonic wall is exposed and therefore more susceptible to other pathogenetic factors.

\section{Low Fiber Intake}

The major traditional hypothesis concerning the propensity to form diverticula addresses the consumption of dietary fiber [4-6]. Data from both clinical studies and experimental animal models have shown that a low fiber intake positively correlates with the formation of diverticula $[7,8]$. As colonic transit time is delayed by a lack of dietary fibers [9], it has been proposed that a reduction in fiber-induced water absorption leads to smaller amounts of stool and a diminishment in luminal diameter. According to the law of Laplace, a smaller radius will result in higher intraluminal pressure and, hence, facilitate the development of diverticula [4]. However, although dietary fibers have been proven to be protective against these alterations, there is no clear evidence for a benefit of a high fiber intake in asymptomatic diverticular disease to prevent the onset of symptoms [8].

\section{Alterations in Connective Tissue}

Like many other organs, with increasing age the intestine is subject to altered metabolism of extracellular matrix components. The corresponding studies have focused on structural alterations of the connective tissue. Two major extracellular matrix components, collagen and elastin, have been found to be altered in diverticular disease: smaller, more densely packed collagen fibrils and an excessive cross-linking of collagen molecules with increased rigidity and a subsequent loss of tensile strength $[10,11]$ and an increase of elastic fibers (elastosis) confined to the longitudinal muscle layer with a subsequent thickening of the colonic wall [12]. The higher rigidity of the colon is due to reduced reset force more susceptible to the formation of diverticula. In addition, more recent data have shown that matrix metalloproteinases or their inhibitors responsible for collagen metabolism are altered in patients with diverticular disease $[13,14]$.

\section{Novel Pathogenetic Concepts}

Alterations of Intestinal Motility in Diverticular Disease

The most accepted pathophysiologic common final pathway resulting in mucosal herniation appears to be an increased intraluminal pressure. It is thought that excessive segmental contractions transform the colonic wall into concertina- or bladder-like compartments ('bladder colon') causing both a functional obstruction and painful sensations. Evidence for disturbed motility patterns is mainly derived from manometric and myoelectric studies showing that patients with diverticular disease, particularly those with a symptomatic course, are characterized by higher intracolonic pressure and increased motility indices after administration of meals or provocative pharmacologic agents such as morphine or prostigmine [15-17]. As most of the early investigations were biased by inconsistent methodological approaches (e.g. patient selection, anatomical site of recording, time period of recording), other studies have focused on those colonic segments truly affected by diverticula $[18,19]$ and have prolonged the recording time [20] revealing that diverticular disease is associated with an increased motor activity and high-amplitude propagated contractions.

\section{Enteric Neuromuscular Pathology: An Underlying \\ Mechanism for Diverticular Disease?}

Intact intestinal peristalsis requires the precise interaction of all key-components regulating intestinal motility, namely the enteric nervous system ('initiators'), enteric neurotransmitters and their receptors ('mediators'), and the enteric musculature ('effectors'). Consequently, defects in one or several of these components would result in altered colonic motility and thus could account for the development of diverticula.

Recently, the 'London classification of gastrointestinal neuromuscular pathology' defined an enteric neuropathy as a histopathology of the enteric nervous system (ENS) associated with motility disorders of the gastrointestinal tract [21]. According to these guidelines, colonic motility disorders such as congenital and acquired megacolon, slow-transit constipation, and chronic intestinal pseudoobstruction could be linked to defined enteric neuropathies raising the question whether diverticular disease may also fulfil these criteria.

\section{Alterations of the Initiators of Intestinal Motility:}

Enteric Neuropathy in Diverticular Disease

Dating back to 1965, Macbeth and Hawthorn [22] first described an 'acquired neuromuscular derangement' 
characterized by ectopic and enlarged myenteric ganglia due to an increase in glial cells. However, it took two decades until this attractive pathogenetic concept was picked up again and re-evaluated by quantifying studies of the ENS.

Whereas Vuong et al. [23] could not find relevant alterations of the ENS, other studies in patients with diverticular disease were able to confirm a significant decrease in enteric nerve cell density [24, 25], glial cell tissue [26], and intramuscular nerve fiber density [27]. Stoss and Meier-Ruge [28] have primarily focused on the submucosal plexus and described hypertrophied nerve fiber strands and so-called submucosal giant ganglia, both features resembling what has been defined by Meier-Ruge as intestinal neuronal dysplasia [29].

We recently evaluated morphological changes of the ENS according to the guidelines of the London classification using $\mathrm{HuC} / \mathrm{D}$ as a pan-neuronal marker. This antigen is restricted to neuronal somata enabling cell-by-cell counts unaffected by co-staining of neuronal processes. The systematic quantitation of enteric nerve and glial cells revealed a reduced neuronal density in all enteric nerve plexus and a decrease of ganglionic nerve cell content in the myenteric plexus corresponding to an oligoneuronal hypoganglionosis [30]. In addition, the loss of enteric neurons was accompanied by decreased myenteric glial cell density.

\section{Alterations of the Effectors of Intestinal Motility:}

Enteric Myopathy in Diverticular Disease

Patients with diverticular disease often show a peculiar thickening of the tunica muscularis affecting both the circular and longitudinal muscle layers [31]. This finding does not appear to result from hyperplasia of the muscle but instead was rather ascribed to architecturally altered myocytes [32]. In addition, augmented presence of elastin has been described [12]. These changes affect mainly the longitudinal muscle layer, whereas the circular muscle shows normal elastin contents. The subsequent shortening of the bowel tube leads to a concertinalike folding and an excess of mucosal tissue susceptible to be pushed through loci minoris resistantiae of the colonic wall. Based on the pathophysiological concept raised by Painter and Burkitt [4], the longitudinal contraction of the bowel wall results in hypersegmentation and the formation of a bladder-colon. Within these compartments intraluminal pressures of up to $90 \mathrm{~mm} \mathrm{Hg}$ may develop giving rise to pulsatile pseudodiverticula.

By evaluating Azan-stained sections of the colonic wall we observed massive intramuscular fibrosis in a sub- set of patients with diverticular disease. The fibrotic transformation affected both muscle layers arguing for a degenerative process of the enteric musculature in diverticular disease [Wedel et al., unpubl. observations]. More recently, we investigated mRNA expression profiles of several components of the contractile apparatus of smooth muscle cells by real-time PCR experiments of the tunica muscularis obtained from patients with diverticular disease. mRNA expression of tropomyosin and caldesmon was specifically downregulated in patients with diverticular disease compared to controls [Böttner et al., unpubl. obs.], indicating that the enteric musculature is not only compromised at morphological level but also affected by reduced gene expression of proteins essential for the functional integrity.

\section{Alterations of the Mediators of Intestinal Motility:}

Disturbed Neurotransmitter Systems in Diverticular

\section{Disease}

The potentially disturbed enteric neuromuscular communication has been proven by demonstrating alterations in several enteric neurotransmitter systems: Milner et al. [33] have found an increased level of the neurotransmitter vasoactive intestinal polypeptide in patients with diverticular disease, Golder et al. [34] described lower nitric oxide immunoreactivity in the longitudinal muscle, and Simpson et al. [35] demonstrated significantly higher levels of substance P, neuropeptide K, PACAP, galanin and VIP within the mucosal plexus. However, these nonuniform and partly contradictory findings indicate that there is no clear evidence that changes in one single neurotransmitter might account for the disturbed intestinal motility patterns observed in patients with diverticular disease.

Thus, an alternative approach to study disturbances in enteric neurotransmitter systems turned out to aim at focusing at the receiving end of neurotransmitters, namely to address changes in the corresponding receptors. Along this line, Golder et al. [27] have investigated the role of smooth muscle and neural cholinergic activity in diverticular disease. The authors described upregulation of smooth muscle muscarinergic M3 receptors concomitantly with an increased sensitivity to exogenous acetylcholine. Recent data from our laboratory demonstrate that within the serotonergic system the serotonin 4 receptor is specifically downregulated in the tunica muscularis of patients with diverticular disease [Böttner et al., unpubl. obs.]. Since this receptor exerts excitatory functions within the ENS [36] and mediates relaxation of the smooth muscle [37] this finding may 
help to explain the disturbed intestinal motor patterns frequently observed in patients with diverticular disease.

Alterations of Neurotrophic Factors: Role of Glial Cell Line-Derived Neurotrophic Factor in Diverticular

Disease

Glial cell line-derived neurotrophic factor (GDNF) is a crucial signaling molecule regulating the development and survival of enteric neurons. Its name is based on the fact that the molecule was initially isolated from the supernatant of a glial cell line and characterized by its ability to promote the survival of cultured dopaminergic neurons [38]. GDNF is a member of the transforming growth factor superfamily of growth factors which exert various functions in the regulation of the development and function of the nervous system [39]. The GDNFinduced signal transduction is mediated by a glycosylphosphatidylinositol-coupled GDNF-family receptor- $\alpha 1$ (GFR- $\alpha 1$ ) and the tyrosin kinase receptor RET [40]. Loss of GDNF leads to total aganglionosis in the small intestine and colon of mutant mice [40,41]. A similar gross phenotype is displayed by knock-out mutants in which the genes for GFR $\alpha 1$ or RET have been deleted [42, 43]. Furthermore, RET mutations account for $50 \%$ of familial forms of Hirschsprung's disease [44].

Although in diverticular disease the colonic wall does not display aganglionosis (complete loss of enteric nerve cells), we recently demonstrated that the ENS is characterized by hypoganglionosis (partial loss of enteric nerve cells) [30] and thus addressed the question whether the GDNF system may be compromised in diverticular disease. By means of a low-density PCR array, we found that indeed GDNF mRNA expression was downregulated in the tunica muscularis of patients with diverticular disease compared to controls [Böttner et al., unpubl. obs.]. Furthermore, in vitro experiments have shown that GDNF acts as a neurotrophic factor promoting the survival and differentiation of cultured postnatal myenteric neurons. Taking these findings together, we hypothesize that the observed loss of enteric nerve cells in diverticular disease may be attributed at least in part to a deficit of GDNF and to a subsequent shortfall of its neurotrophic potential on enteric neurons.

\section{Role of Postinflammatory Alterations of the ENS in Diverticular Disease}

Depending upon the degree and duration of inflammation, the mucosa and adjacent tissue layers frequently display persistent histological alterations following an acute phase of diverticulitis. As summarized by Brian West et al. [45] increased lymphoplasmacytic infiltrates are observed in the lamina propria associated with mucin depletion, cryptitis, architectural distortion, Paneth cell metaplasia, and the formation of lymphoglandular complexes. As the ENS extends throughout all the intestinal layers, it is conceivable that both enteric nerve fibers and ganglia are affected by postinflammatory alterations in particular in chronic stages of diverticular disease. In fact, Simpson et al. [35] have described an increase of nerve fibers in the mucosa as well as in the tunica muscularis of patients with diverticular disease accompanied by upregulation of pain-mediating peptides, e.g. substance $P$, neuropeptide $\mathrm{K}$ and galanin. It has been suggested that this proliferative nerve remodeling could be considered as a form of regenerative hyperinnervation leading to increased visceral hypersensitivity and thus the generation of persistent symptoms in diverticular disease.

\section{Conclusions}

The remaining question that cannot be answered at this point is whether the neuromuscular alterations observed in diverticular disease correspond to a primary or secondary event - are they hen or egg? If the enteric neuromuscular pathology reflects a primary lesion, this could in turn lead to disturbed intestinal motility pattern, increased intraluminal pressure and ultimately to the formation of diverticula. If inflammatory events are considered as the driving force for the neuromuscular abnormalities, these changes would arise as secondary lesions resembling an associated pathology.

It seems conceivable that both concepts may hold true in diverticular disease, as on the one hand myenteric nerve cell loss has also been reported in patients with 'cold diverticula' speaking in favor of a primary enteric neuropathy [24], and on the other hand the development of postinflammatory alterations of the ENS have been proven also in other conditions such as in postinfectious irritable bowel syndrome. Therefore, the impact of the aforementioned spectrum of enteric neuromuscular pathologies should deserve further attention in regards to both the pathogenesis of and the generation of symptoms in diverticular disease.

\section{Disclosure Statement}

The authors declare that no financial or other conflict of interest exists in relation to the content of the article. 


\section{References}

1 Sandler RS, et al: The burden of selected digestive diseases in the United States. Gastroenterology 2002;122:1500-1511.

2 Parks TG: Post-mortem studies on the colon with special reference to diverticular disease. Proc R Soc Med 1968;61:932-934.

-3 Hughes LE: Postmortem survey of diverticular disease of the colon. II. The muscular abnormality of the sigmoid colon. Gut 1969;10: 344-351.

4 Painter NS, Burkitt DP: Diverticular disease of the colon, a 20th century problem. Clin Gastroenterol 1975;4:3-21.

5 Miura S, et al: Recent trends in diverticulosis of the right colon in Japan: retrospective review in a regional hospital. Dis Colon Rectum 2000;43:1383-1389.

6 Painter NS: The cause of diverticular disease of the colon, its symptoms and its complications. Review and hypothesis. J R Coll Surg Edinb 1985;30:118-122.

7 Gear JS, et al: Symptomless diverticular disease and intake of dietary fibre. Lancet 1979;i:511-514.

8 Wess L, et al: An association between maternal diet and colonic diverticulosis in an animal model. Gut 1996;39:423-427.

9 Burkitt DP, Walker AR, Painter NS: Effect of dietary fibre on stools and the transit-times, and its role in the causation of disease. Lancet 1972;ii:1408-1412.

10 Wess L, et al: Cross linking of collagen is increased in colonic diverticulosis. Gut 1995; 37:91-94.

11 Thomson HJ, et al: The submucosa of the human colon. J Ultrastruct Mol Struct Res 1986;96:22-30.

12 Whiteway J, Morson BC: Elastosis in diverticular disease of the sigmoid colon. Gut $1985 ; 26: 258-266$

13 Stumpf M, et al: Increased distribution of collagen type III and reduced expression of matrix metalloproteinase 1 in patients with diverticular disease. Int $\mathrm{J}$ Colorectal Dis 2001;16:271-275.

14 Mimura T, et al: Up-regulation of collagen and tissue inhibitors of matrix metalloproteinase in colonic diverticular disease. Dis Colon Rectum 2004;47:371-378; discussion 378-379.

15 Arfwidsson S, et al: Pathogenesis of multiple diverticula of the sigmoid colon in diverticular disease. Acta Chir Scand 1964;63:(suppl 342):1-68.

16 Painter NS, et al: Effect of morphine, prostigmine, pethidine, and probanthine on the human colon in diverticulosis studied by intraluminal pressure recording and cineradiography. Gut 1965;6:57-63.
17 Weinreich J, Andersen D: Intraluminal pressure in the sigmoid colon. II. Patients with sigmoid diverticula and related conditions. Scand J Gastroenterol 1976;11:581-586.

18 Trotman IF, Misiewicz JJ: Sigmoid motility in diverticular disease and the irritable bowel syndrome. Gut 1988;29:218-222.

19 Cortesini C, Pantalone D: Usefulness of colonic motility study in identifying patients at risk for complicated diverticular disease. Dis Colon Rectum 1991;34:339-342.

20 Bassotti G, et al: Twenty-four hour recordings of colonic motility in patients with diverticular disease: evidence for abnormal motility and propulsive activity. Dis Colon Rectum 2001;44:1814-1820.

21 Knowles CH, et al: The London Classification of gastrointestinal neuromuscular pathology: report on behalf of the Gastro 2009 International Working Group. Gut 2010;59: 882-887.

22 Macbeth WA, Hawthorne JH: Intramural ganglia in diverticular disease of the colon. J Clin Pathol 1965;18:40-42.

23 Vuong NP, et al: Myenteric plexuses and colonic diverticulosis: results of a histological study. Gastroenterol Clin Biol 1985;9:434436.

24 Iwase $\mathrm{H}$, et al: Morphology of myenteric plexuses in the human large intestine: comparison between large intestines with and without colonic diverticula. J Clin Gastroenterol 2005;39:674-678

25 Deduchovas O, et al: Morphologic pattern of myenteric neural plexus in colonic diverticular disease. A whole-mount study employing histochemical staining for acetylcholinesterase. Ann Anat 2008;190:525-530.

26 Bassotti G, et al: Interstitial cells of Cajal, enteric nerves, and glial cells in colonic diverticular disease. J Clin Pathol 2005;58:973977.

27 Golder M, et al: Smooth muscle cholinergic denervation hypersensitivity in diverticular disease. Lancet 2003;361:1945-1951.

28 Stoss F, Meier-Ruge W: Diagnosis of neuronal colonic dysplasia in primary chronic constipation and sigmoid diverticulosis endoscopic biopsy and enzyme-histochemical examination. Surg Endosc 1991;5:146-149.

29 Meier-Ruge W, et al: The neuropathological diagnosis of neuronal intestinal dysplasia (NID B). Eur J Pediatr Surg 1994;4:267-273.

-30 Wedel T, et al: Diverticular disease is associated with an enteric neuropathy as revealed by morphometric analysis. Neurogastroenterol Motil 2010;22:407-414, e93-e94.

31 Morson BC: The muscle abnormality in diverticular disease of the colon. Proc R Soc Med 1963;56:798-800.
32 Raguse T, Kuhnel W: Pathogenesis of colon diverticular disease (author's transl). Leber Magen Darm 1981;11:147-158.

- 33 Milner P, et al: Vasoactive intestinal polypeptide levels in sigmoid colon in idiopathic constipation and diverticular disease. Gastroenterology 1990;99:666-675.

34 Golder M, et al: Longitudinal muscle shows abnormal relaxation responses to nitric oxide and contains altered levels of NOS1 and elastin in uncomplicated diverticular disease. Colorectal Dis 2007;9:218-228.

- 35 Simpson J, et al: Post inflammatory damage to the enteric nervous system in diverticular disease and its relationship to symptoms. Neurogastroenterol Motil 2009;21:847e858.

36 Gershon MD: Review article: serotonin receptors and transporters - roles in normal and abnormal gastrointestinal motility. Aliment Pharmacol Ther 2004;20(suppl 7):314.

37 Kuemmerle JF, et al: Coexpression of 5-HT2A and 5-HT4 receptors coupled to distinct signaling pathways in human intestinal muscle cells. Gastroenterology 1995;109: 1791-1800.

38 Lin LF, et al: Purification and initial characterization of rat B49 glial cell line-derived neurotrophic factor. J Neurochem 1994;63: 758-768.

39 Bottner M, Krieglstein K, Unsicker K: The transforming growth factor-betas: structure, signaling, and roles in nervous system development and functions. J Neurochem 2000;75:2227-2240.

40 Airaksinen MS, Saarma M: The GDNF family: signalling, biological functions and therapeutic value. Nat Rev Neurosci 2002;3:383394

41 Moore MW, et al: Renal and neuronal abnormalities in mice lacking GDNF. Nature 1996; 382:76-79.

42 Durbec P, et al: GDNF signalling through the Ret receptor tyrosine kinase. Nature 1996; 381:789-793

43 Cacalano G, et al: GFRalpha1 is an essential receptor component for GDNF in the developing nervous system and kidney. Neuron 1998;21:53-62.

44 Attie T, et al: Diversity of RET proto-oncogene mutations in familial and sporadic Hirschsprung disease. Hum Mol Genet 1995;4:1381-1386.

45 Brian West A: The pathology of diverticulosis: classical concepts and mucosal changes in diverticula. J Clin Gastroenterol 2006; 40(suppl 3):S126-S131. 